\title{
VERTEX ALGEBRAS ACCORDING TO ISAAC NEWTON
}

\author{
MICHAEL TUITE
}

\begin{abstract}
We give an introduction to vertex algebras using elementary forward difference methods originally due to Isaac Newton.
\end{abstract}

\section{INTRODUCTION}

In this paper we present an introduction to the theory of vertex algebras $[B]$, $[\overline{\mathrm{FLM}}],[\overline{\mathrm{K}}],[\overline{\mathrm{LL}}],[\overline{\mathrm{FHL}}],[\mathrm{LZ}],[\mathrm{MN}],[\overline{\mathrm{MT}}]$. A cursory examination of the literature of vertex algebras reveals a variety of identities involving binomial coefficients

$$
\left(\begin{array}{c}
n \\
i
\end{array}\right)=\frac{n(n-1) \ldots(n-i+1)}{i !},
$$

for all $n \in \mathbb{Z}$. We describe how all of these arise from Newton's binomial theorem either directly or else through elementary Newton finite difference identities [N]. In particular, our approach provides both a motivation and a new understanding of the fundamental axioms of locality and lower truncation for vertex operators. We also obtain a simplified and stronger proof of the Borcherds-Frenkel-Lepowsky-Meurmann identity.

\section{NeWTON ForWARd Differences AND Formal SERIES}

2.1. Forward Differences. We consider an elementary but very relevant illustration of formal series techniques used in vertex algebra theory. Our example comes from Newton's theory of finite differences.]

Let $U$ be a vector space over a field of characteristic zero. Let $U^{\mathbb{Z}}$ denote the set of doubly infinite sequences $\alpha=\left\{\alpha_{n}\right\}_{n \in \mathbb{Z}}$ with components $\alpha_{n} \in U$. Define the (first) forward difference operator $\Delta: U^{\mathbb{Z}} \rightarrow U^{\mathbb{Z}}$

$$
(\Delta \alpha)_{n}=\alpha_{n+1}-\alpha_{n}, \quad \alpha \in U^{\mathbb{Z}} .
$$

The $N^{\text {th }}$ forward difference operator is defined for all integers $N \geq 2$ by

$$
\Delta^{N}=\Delta \circ \Delta^{N-1} .
$$

Example 1. For a real function $f(x)$ define $\alpha_{n}=f(n) \in \mathbb{R}$. Then $(\Delta \alpha)_{n}$ is the classical Newton forward difference used in the polynomial interpolation of $f(x)$.

The action of $\Delta^{N}$ on $U^{\mathbb{Z}}$ is given by:

Lemma 2. The $N^{\text {th }}$ forward difference of $\alpha \in U^{\mathbb{Z}}$ has components

$$
\left(\Delta^{N} \alpha\right)_{n}=\sum_{i \geq 0}(-1)^{i}\left(\begin{array}{c}
N \\
i
\end{array}\right) \alpha_{n+N-i}
$$

Key words and phrases. Vertex Algebras, Conformal Field Theory, Finite Differences.

${ }^{1}$ Borcherds [B] defined vertex algebras whilst at Trinity College Cambridge exactly 300 years after Newton $[\mathbb{N}]$ invented finite differences at the same institution! 
Proof. Write $\Delta=F-I$ where $F$ is the forward shift operator

$$
(F \alpha)_{n}=\alpha_{n+1},
$$

and $I$ is the identity operator. The result follows from Newton's binomial identity

$$
(F-I)^{N} \alpha=\sum_{i \geq 0}\left(\begin{array}{c}
N \\
i
\end{array}\right)(-1)^{i} F^{N-i} \alpha .
$$

We now consider ker $\Delta^{N}$, the space of sequences with zero $N^{\text {th }}$ forward difference.

Proposition 3 (Newton's Forward Difference Formula). Let $\alpha \in U^{\mathbb{Z}}$ with components $\alpha_{n}$. If $\alpha \in \operatorname{ker} \Delta^{N}$ for some $N \geq 1$ then for all $n \in \mathbb{Z}$

$$
\alpha_{n}=\sum_{i \geq 0}\left(\begin{array}{c}
n \\
i
\end{array}\right)\left(\Delta^{i} \alpha\right)_{0} .
$$

Conversely, if $\alpha \in U^{\mathbb{Z}}$ has components $\alpha_{n}=\sum_{i=0}^{N-1}\left(\begin{array}{c}n \\ i\end{array}\right) R_{i}$ for $R_{i} \in U$ and some $N \geq 1$ then $\alpha \in \operatorname{ker} \Delta^{N}$.

Proof. Assume $\alpha \in \operatorname{ker} \Delta^{N}$. We have $\alpha_{n}=\left(F^{n} \alpha\right)_{0}$ for all $n \in \mathbb{Z}$ with $F$ the forward shift operator of (3). Then (4) follows from a binomial expansion of $F^{n} \alpha=(I+\Delta)^{n} \alpha$

$$
F^{n} \alpha=\sum_{i \geq 0}\left(\begin{array}{c}
n \\
i
\end{array}\right) \Delta^{i} \alpha
$$

for all $n \in \mathbb{Z}$. For $n \geq 0$, (5) is obvious whereas for $n=-k<0$ we can verify that

$$
\alpha=F^{k} \sum_{i \geq 0}\left(\begin{array}{c}
-k \\
i
\end{array}\right) \Delta^{i} \alpha,
$$

for all $\alpha \in \operatorname{ker} \Delta^{N}$. Hence (5) holds and therefore (4) results.

Conversely, if $\alpha_{n}=\sum_{i=0}^{N-1}\left(\begin{array}{c}n \\ i\end{array}\right) R_{i}$ then noting that for $\beta_{n}=n^{k}$ and $k>0$

$$
(\Delta \beta)_{n}=k n^{k-1}+O\left(n^{k-2}\right),
$$

we find $\alpha \in \operatorname{ker} \Delta^{N}$ since $\left(\begin{array}{c}n \\ i\end{array}\right)=\frac{1}{i !} n^{i}+O\left(n^{i-1}\right)$.

We also note the following result:

Corollary 4. $\alpha \in \operatorname{ker} \Delta^{N}$ iff $\alpha_{n}=p_{N-1}(n)$ where $p_{N-1}(n)$ is a degree $N-1$ polynomial in $n$ with coefficients in $U$.

Example 5. Let $p_{N-1}(x)$ be a polynomial of degree $N-1$ with coefficients in $\mathbb{R}$. Then Proposition 3 is Newton's forward difference formula expressing $p_{N-1}(n)$ for all $n \in \mathbb{Z}$ in terms of $p_{N-1}(i)$ for $i=0, \ldots, N-1$. Replacing $n$ by $x$ on the right hand side of (4) gives the Newton interpolating polynomial for a real function $f(x)$ in terms of $\alpha_{i}=f(i)$ for $i=0,1, \ldots, N-1$. 
2.2. Formal Generating Series. Define a formal generating series $\alpha(z)$ for $\alpha \in U^{\mathbb{Z}}$ by

$$
\alpha(z)=\sum_{n \in \mathbb{Z}} \alpha_{n} z^{-n-1} \in U\left[\left[z, z^{-1}\right]\right],
$$

where $U\left[\left[z, z^{-1}\right]\right]$ denotes the space of formal Laurent series in an indeterminate parameter $z$ with coefficients in $U$. We associate the component $\alpha_{n}$ with $z^{-n-1}$ for reasons that become clearer below e.g. (16), Lemma 8 and Theorem 24.

Define the formal derivative $\partial$ of $\alpha(z)$ by

$$
\partial \alpha(z)=\sum_{n \in \mathbb{Z}} \alpha_{n}(-n-1) z^{-n-2}=\sum_{n \in \mathbb{Z}}\left(-n \alpha_{n-1}\right) z^{-n-1} .
$$

We also define $\partial^{(i)}:=\frac{1}{i !} \partial^{i}$. The formal residue of the Laurent series (6) is defined by

$$
\operatorname{Res}_{z} \alpha(z)=\alpha_{0} .
$$

Lemma 6. The formal series $\alpha(z)$ satisfies versions of the fundamental theorem of calculus, the Leibniz rule and integration by parts:

$$
\begin{aligned}
& \operatorname{Res}_{z} \partial \alpha(z)=0, \\
& \partial\left(z^{k} \alpha(z)\right)=k z^{k-1} \alpha(z)+z^{k} \partial \alpha(z), \\
& \operatorname{Res}_{z} z^{k} \partial \alpha(z)=-k \operatorname{Res}_{z} z^{k-1} \alpha(z),
\end{aligned}
$$

for all $k \in \mathbb{Z}$.

Proof. (7) immediately implies (9) and (10). $\operatorname{Res}_{z} \partial\left(z^{k} \alpha(z)\right)=0$ implies (11).

The formal nature of a series $\alpha(z)$ is well-illustrated by the constant sequence $\alpha_{n}=\alpha_{0} \in U$, for all $n$, for which

$$
\alpha(z)=\alpha_{0} \delta(z)
$$

for formal delta series defined by

$$
\delta(z)=\sum_{m \in \mathbb{Z}} z^{m} .
$$

The delta series is analogous to the Dirac delta function in the sense that

$$
z^{k} \delta(z)=\delta(z)
$$

for all $k \in \mathbb{Z}$. In particular, we note that

$$
(z-1) \delta(z)=0 .
$$

We also define a family of formal delta series indexed by integers $i \geq 0$ as follows:

$$
\delta^{(i)}(z)=(-1)^{i} \partial^{(i)} \delta(z)=\sum_{m \in \mathbb{Z}}(-1)^{i}\left(\begin{array}{c}
m \\
i
\end{array}\right) z^{m-i},
$$

with $\delta^{(0)}(z)=\delta(z)$. On relabelling, we note that (15) can be rewritten as

$$
\delta^{(i)}(z)=\sum_{n \in \mathbb{Z}}\left(\begin{array}{c}
n \\
i
\end{array}\right) z^{-n-1},
$$

i.e. $\delta^{(i)}(z)$ is the formal series for the integer sequence $\left\{\left(\begin{array}{c}n \\ i\end{array}\right)\right\}_{n \in \mathbb{Z}}$. We further find that (14) generalises to:

Lemma 7. $(z-1) \delta^{(i)}(z)=\delta^{(i-1)}(z)$ for all $i \geq 1$. 
Since $(F \alpha)(z)=z \alpha(z)$ for the forward shift operator of (3), it follows that the formal series for $\Delta^{i} \alpha$ is

$$
\left(\Delta^{i} \alpha\right)(z)=(z-1)^{i} \alpha(z) .
$$

Thus $\alpha \in \operatorname{ker} \Delta^{N}$ iff $(z-1)^{N} \alpha(z)=0$. Noting that

$$
\left(\Delta^{i} \alpha\right)_{0}=\operatorname{Res}_{z}(z-1)^{i} \alpha(z),
$$

we may reformulate Newton's forward difference formula Proposition 3 in terms of formal series using (15) and (18) to find:

Lemma 8. Let $\alpha \in U^{\mathbb{Z}}$. Then $\alpha \in \operatorname{ker} \Delta^{N}$ iff

$$
\alpha(z)=\sum_{i=0}^{N-1} R_{i} \delta^{(i)}(z)
$$

for $R_{i}=\operatorname{Res}_{z}(z-1)^{i} \alpha(z) \in U$.

In numerous classical applications of generating series with $U=\mathbb{C}$, the formal parameter $z$ can be taken to be a complex number in some domain on which the generating series converges. However, the formal delta series $\delta^{(i)}(z)$ diverges everywhere on the complex plane. Nevertheless, if we decompose $\delta^{(i)}(z)=\delta^{(i)}(z)_{+}+\delta^{(i)}(z)_{-}$with

$$
\delta^{(i)}(z)_{+}=\sum_{n \geq 0}\left(\begin{array}{c}
n \\
i
\end{array}\right) z^{-n-1}, \quad \delta^{(i)}(z)_{-}=\sum_{n \leq-1}\left(\begin{array}{c}
n \\
i
\end{array}\right) z^{-n-1},
$$

where the \pm subscripts refer to the sign of the sequence index $n$. Then the series $\delta^{(i)}(z)_{+}$and $\delta^{(i)}(z)_{-}$converge on disjoint complex domains as follows:

$$
\delta^{(i)}(z)_{+}=\frac{1}{(z-1)^{i+1}}, \quad|z|>1, \quad \delta^{(i)}(z)_{-}=\frac{-1}{(z-1)^{i+1}}, \quad|z|<1 .
$$

We utilise these expansions for formal $z$ by adopting the following convention:

Definition 9 (Expansion Convention). For $m \in \mathbb{Z}$ and formal variables $x, y$ we define

$$
(x+y)^{m}=\sum_{k \geq 0}\left(\begin{array}{c}
m \\
k
\end{array}\right) x^{m-k} y^{k},
$$

i.e. we expand in the second variable. For $m \geq 0,(x+y)^{m}=(y+x)^{m}$, with a finite sum, whereas for $m<0,(x+y)^{m}$ and $(y+x)^{m}$ are distinct infinite series.

Following this convention we write

$$
\delta^{(i)}(z)_{+}=\frac{1}{(z-1)^{i+1}}, \quad \delta^{(i)}(z)_{-}=-\frac{1}{(-1+z)^{i+1}},
$$

so that

$$
\delta^{(i)}(z)=\frac{1}{(z-1)^{i+1}}-\frac{1}{(-1+z)^{i+1}} .
$$

We may similarly decompose any formal series as $\alpha(z)=\alpha(z)_{+}+\alpha(z)_{-}$where

$$
\alpha(z)_{+}=\sum_{n \geq 0} \alpha_{n} z^{-n-1}, \quad \alpha(z)_{-}=\sum_{n \leq-1} \alpha_{n} z^{-n-1} .
$$


The \pm subscripts refer to the sign of the sequence index $n$ 2 Thus Lemma 8 and (22) imply $\alpha \in \operatorname{ker}\left(\Delta^{N}\right)$ iff

$$
\alpha(z)_{+}=\sum_{i=0}^{N-1} \frac{R_{i}}{(z-1)^{i+1}}, \quad \alpha(z)_{-}=-\sum_{i=0}^{N-1} \frac{R_{i}}{(-1+z)^{i+1}} .
$$

Altogether the following theorem summarises our discussion thus far.

Theorem 10. Let $\alpha \in U^{\mathbb{Z}}$ with formal series $\alpha(z)$ and let

$$
R_{i}=\left(\Delta^{i} \alpha\right)_{0}=\operatorname{Res}_{z}(z-1)^{i} \alpha(z) \in U,
$$

for integers $i \geq 0$. The following are equivalent:

(i) $\alpha \in \operatorname{ker} \Delta^{N}$,

(ii) $(z-1)^{N} \alpha(z)=0$,

(iii) $\alpha_{n}=\sum_{i=0}^{N-1}\left(\begin{array}{c}n \\ i\end{array}\right) R_{i}$ for all $n \in \mathbb{Z}$,

(iv) $\alpha_{n}=p_{N-1}(n)$, a degree $N-1$ polynomial in $n$ with coefficients in $U$,

(v) $\alpha(z)=\sum_{i=0}^{N-1} R_{i} \delta^{(i)}(z)$

(vi) $\alpha(z)_{+}=\sum_{i=0}^{N-1} \frac{R_{i}}{(z-1)^{i+1}}$ and $\alpha(z)_{-}=-\sum_{i=0}^{N-1} \frac{R_{i}}{(-1+z)^{i+1}}$.

2.3. Calculus of Formal Series. We gather a compendium of results concerning formal series that we make use of later.

Lemma 11 (Taylor's Theorem). For formal series $\alpha(z)$ then $\alpha(x+y)$ has formal Taylor expansion in $y$

$$
\alpha(x+y)=e^{y \partial} \alpha(x)
$$

where $e^{y \partial}:=\sum_{i \geq 0} y^{i} \partial^{(i)}$.

Proof. Using the formal expansion convention Definition 9 we find

$$
\begin{aligned}
\alpha(x+y) & =\sum_{n \in \mathbb{Z}} \alpha_{n}(x+y)^{-n-1}=\sum_{n \in \mathbb{Z}} \alpha_{n} \sum_{i \geq 0}\left(\begin{array}{c}
-n-1 \\
i
\end{array}\right) x^{-n-1-i} y^{i} \\
& =\sum_{n \in \mathbb{Z}} \alpha_{n} \sum_{i \geq 0} y^{i} \partial^{(i)}\left(x^{-n-1}\right)=\sum_{i \geq 0} y^{i} \partial^{(i)} \alpha(x) .
\end{aligned}
$$

Lemma 12. For $\alpha(z)_{ \pm}$of (23) we have

$$
\partial\left(\alpha(z)_{ \pm}\right)=(\partial \alpha(z))_{ \pm} .
$$

Lemma 13 (Residue Theorem). Let $\alpha(z)$ be a formal series. For integer $k \geq 0$ we have

$$
\operatorname{Res}_{x} \frac{\alpha(x)}{(x-z)^{k+1}}=\partial^{(k)} \alpha(z)_{-}, \quad \operatorname{Res}_{x} \frac{\alpha(x)}{(-z+x)^{k+1}}=-\partial^{(k)} \alpha(z)_{+} .
$$

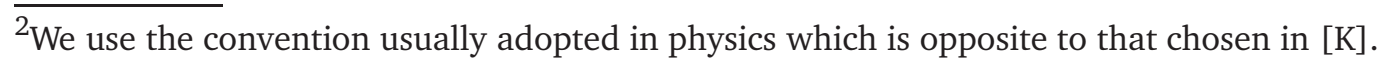


Proof. Consider

$$
\operatorname{Res}_{x} \frac{\alpha(x)}{x-z}=\operatorname{Res}_{x} \sum_{n \in \mathbb{Z}} \sum_{r \geq 0} \alpha_{n} x^{-n-r-2} z^{r}=\sum_{r \geq 0} \alpha_{-r-1} z^{r}=\alpha(z)_{-} .
$$

Similarly we find $\operatorname{Res}_{x} \frac{\alpha(x)}{-z+x}=-\alpha(z)_{+}$. Thus (26) holds for $k=0$. The general result follows on applying $\partial_{z}^{(k)}$ and using (25).

\section{LOCALITY}

3.1. Locality of Formal Series. Let us now assume that $U$ is an associative algebra over a field of characteristic zero i.e. $U$ is a vector space equipped with an associative bilinear product $A B \in U$ for all $A, B \in U$. In the next section we will consider $U$ to be the algebra of endomorphisms of a vector space $V$.

We define the formal product and commutator for formal generating series $\alpha(z), \beta(z) \in U\left[\left[z, z^{-1}\right]\right]$ by

$$
\begin{aligned}
\alpha(x) \beta(y) & =\sum_{m, n \in \mathbb{Z}} \alpha_{m} \beta_{n} x^{-m-1} y^{-n-1} \in U\left[\left[x, x^{-1}, y, y^{-1}\right]\right], \\
{[\alpha(x), \beta(y)] } & =\sum_{m, n \in \mathbb{Z}}\left[\alpha_{m}, \beta_{n}\right] x^{-m-1} y^{-n-1} \in U\left[\left[x, x^{-1}, y, y^{-1}\right]\right],
\end{aligned}
$$

for independent indeterminates $x$ and $y$ and commutator $\left[\alpha_{m}, \beta_{n}\right]=\alpha_{m} \beta_{n}-\beta_{n} \alpha_{m}$. In the language of Chapter 2, the bivariate series (27) and (28) are generating series for doubly indexed sequences $\left\{\alpha_{m} \beta_{n}\right\}_{m, n \in \mathbb{Z}}$ and $\left\{\left[\alpha_{m}, \beta_{n}\right]\right\}_{m, n \in \mathbb{Z}}$, respectively.

We now define the fundamental notion of locality - one of the most important properties enjoyed by vertex operators [Li], [G], [LZ]. For formal series $\alpha(x), \beta(y)$ and integers $n \geq 0$ we define the formal bivariate series 3

$$
C^{n}(\alpha(x), \beta(y))=(x-y)^{n}[\alpha(x), \beta(y)] .
$$

Definition 14 (Locality). $\alpha(z), \beta(z) \in U\left[\left[z, z^{-1}\right]\right]$ are called mutually local if for some integer $n \geq 0$

$$
C^{n}(\alpha(x), \beta(y))=0 .
$$

The order of locality of $\alpha(z)$ and $\beta(z)$ is the least integer $n=N \geq 0$ for which (30) holds, in which case we say that $\alpha(z)$ and $\beta(z)$ are mutually local of order $N$ and write $\alpha(z) \stackrel{N}{\sim} \beta(z)$ (or simply $\alpha(z) \sim \beta(z)$ if $N$ is not specified). We also say that $\alpha(z)$ is local if $\alpha(z) \sim \alpha(z)$.

Lemma 15. If $\alpha(z) \stackrel{N}{\sim} \beta(z)$ then $\partial \alpha(z) \stackrel{N+1}{\sim} \beta(z)$.

Proof. $0=\partial_{x} C^{N+1}(\alpha(x), \beta(y))=N C^{N}(\alpha(x), \beta(y))+C^{N+1}(\partial \alpha(x), \beta(y))$.

We define the $n^{\text {th }}$ residue product $*_{n}$ for $n \geq 0$ of formal series $\alpha(z), \beta(z)$ to be the formal series ${ }^{4}$

$$
\left(\alpha *_{n} \beta\right)(z)=\operatorname{Res}_{x} C^{n}(\alpha(x), \beta(z))=\sum_{k=0}^{n}\left(\begin{array}{l}
n \\
k
\end{array}\right)(-z)^{k}\left[\alpha_{n-k}, \beta(z)\right] .
$$

\footnotetext{
${ }^{3}$ This is a well-defined formal series in $x$ and $y$ since $n \geq 0$.

${ }^{4}$ The $n^{\text {th }}$ residue product is often also notated by $\alpha(z)_{(n)} \beta(z)$ e.g. [K, $[\mathrm{MN}]$.
} 
For $\alpha(z) \stackrel{N}{\sim} \beta(z)$ it follows that

$$
\left(\alpha *_{n} \beta\right)(z)=0 \text { for all } n \geq N .
$$

The locality condition (30) is closely related to Theorem 10 of the last section for an appropriate choice of vector space and sequence. Let $W=U\left[\left[y, y^{-1}\right]\right]$ be the vector space of formal series in $y$ with coefficients in $U$. If $\alpha(z) \stackrel{N}{\sim} \beta(z)$ then

$$
0=y^{1-N} C^{N}(\alpha(x), \beta(y))=(z-1)^{N} \sum_{n \in \mathbb{Z}} \gamma_{n} z^{-n-1},
$$

for $z=\frac{x}{y}=x y^{-1}$ and

$$
\gamma_{n}=y^{-n}\left[\alpha_{n}, \beta(y)\right] .
$$

$\gamma_{n}$ determines a sequence $\gamma \in W^{\mathbb{Z}}$ with formal series

$$
\gamma(z)=\sum_{n \in \mathbb{Z}} \gamma_{n} z^{-n-1}=y[\alpha(y z), \beta(y)],
$$

where $\alpha(y z)=\sum_{n \in \mathbb{Z}} \alpha_{n}(y z)^{-n-1}$. But (33) implies

$$
(z-1)^{N} \gamma(z)=0,
$$

which is Property (ii) of Theorem 10. Therefore $\gamma$ satisfies the equivalent properties following from Newton's forward difference formula. In particular, Theorem [10 (i) implies $\gamma \in \operatorname{ker} \Delta^{N}$ which together with Lemma 2 implies that for all $n \in \mathbb{Z}$

$$
\sum_{i=0}^{N}\left(\begin{array}{c}
N \\
i
\end{array}\right)(-y)^{i}\left[\alpha_{n-i}, \beta(y)\right]=0 \text {. }
$$

Theorem 10 (iii) determines $\gamma$ in terms of the $N$ residues

$$
R_{i}(y)=\operatorname{Res}_{z}(z-1)^{i} \gamma(z)=y^{-i}\left(\alpha *_{i} \beta\right)(y),
$$

for $i^{\text {th }}$ residue product (31) with $0 \leq i \leq N-1$. Thus Theorem 10 (iii) implies locality is equivalent to

$$
\left[\alpha_{m}, \beta(y)\right]=\sum_{i=0}^{N-1}\left(\begin{array}{c}
m \\
i
\end{array}\right) y^{m-i}\left(\alpha *_{i} \beta\right)(y) .
$$

In terms of components, (36) reads

$$
\left[\alpha_{m}, \beta_{n}\right]=\sum_{i=0}^{N-1}\left(\begin{array}{c}
m \\
i
\end{array}\right)\left(\alpha *_{i} \beta\right)_{m+n-i} .
$$

Theorem 10 (iv)-(vi) describe further corresponding properties equivalent to locality. Recalling (23), Theorem 10 (vi) implies

$$
\left[\alpha(x)_{+}, \beta(y)\right]=\sum_{i=0}^{N-1} \frac{\left(\alpha *_{i} \beta\right)(y)}{(x-y)^{i+1}}, \quad\left[\alpha(x)_{-}, \beta(y)\right]=-\sum_{i=0}^{N-1} \frac{\left(\alpha *_{i} \beta\right)(y)}{(-y+x)^{i+1}},
$$

employing the formal expansion convention (21). Altogether we therefore find Theorem 10 implies the following list of properties equivalent to locality $[\mathrm{K}]$ : 
Theorem 16. Let $\alpha(z), \beta(z) \in U\left[\left[z, z^{-1}\right]\right]$ and let $\left(\alpha *_{i} \beta\right)(z)$ be the $i^{\text {th }}$ residue product for $i \geq 0$. The following are equivalent:

(i) $\alpha(z) \stackrel{N}{\sim} \beta(z)$,

(ii) $\sum_{i=0}^{N}\left(\begin{array}{c}N \\ i\end{array}\right)(-y)^{i}\left[\alpha_{n-i}, \beta(y)\right]=0$ for all $n \in \mathbb{Z}$,

(iii) $\left[\alpha_{m}, \beta(y)\right]=\sum_{i=0}^{N-1}\left(\begin{array}{c}m \\ i\end{array}\right) y^{m-i}\left(\alpha *_{i} \beta\right)(y)$,

(iv) $\left[\alpha_{m}, \beta_{n}\right]=\sum_{i=0}^{N-1}\left(\begin{array}{c}m \\ i\end{array}\right)\left(\alpha *_{i} \beta\right)_{m+n-i}$,

(v) $y^{-m}\left[\alpha_{m}, \beta(y)\right]=p_{N-1}(m)$, where $p_{N-1}(m)$ is a degree $N-1$ polynomial in $m$ with coefficients in $U\left[\left[y, y^{-1}\right]\right]$,

(vi) $[\alpha(x), \beta(y)]=\sum_{i=0}^{N-1} y^{-i-1} \delta^{(i)}\left(\frac{x}{y}\right)\left(\alpha *_{i} \beta\right)(y)$,

(vii) $\left[\alpha(x)_{+}, \beta(y)\right]=\sum_{i=0}^{N-1} \frac{\left(\alpha *_{i} \beta\right)(y)}{(x-y)^{i+1}}$ and $\left[\alpha(x)_{-}, \beta(y)\right]=-\sum_{i=0}^{N-1} \frac{\left(\alpha *_{i} \beta\right)(y)}{(-y+x)^{i+1}}$.

We also define the normally ordered product of $\alpha(x)$ and $\beta(y)$ by

$$
: \alpha(x) \beta(y):=\alpha(x)_{-} \beta(y)+\beta(y) \alpha(x)_{+} .
$$

Thus we find

$$
\begin{aligned}
& \alpha(x) \beta(y)=\left[\alpha(x)_{+}, \beta(y)\right]+: \alpha(x) \beta(y): \\
& \beta(y) \alpha(x)=-\left[\alpha(x)_{-}, \beta(y)\right]+: \alpha(x) \beta(y):
\end{aligned}
$$

which imply

Corollary 17. (OPE) Let $\alpha(z), \beta(z) \in U\left[\left[z, z^{-1}\right]\right]$. Then $\alpha(z) \stackrel{N}{\sim} \beta(z)$ if and only if

$$
\begin{aligned}
& \alpha(x) \beta(y)=\sum_{i=0}^{N-1} \frac{\left(\alpha *_{i} \beta\right)(y)}{(x-y)^{i+1}}+: \alpha(x) \beta(y): \\
& \beta(y) \alpha(x)=\sum_{i=0}^{N-1} \frac{\left(\alpha *_{i} \beta\right)(y)}{(-y+x)^{i+1}}+: \alpha(x) \beta(y): .
\end{aligned}
$$

Remark 18. The above expressions for $\alpha(x) \beta(y)$ and $\beta(y) \alpha(x)$ are related to the Operator Product Expansion (OPE) in chiral conformal field theory (e.g. [BPZ], [FMS])

$$
\alpha(x) \beta(y) \stackrel{\mathrm{OPE}}{\sim} \sum_{i=0}^{N-1} \frac{\left(\alpha *_{i} \beta\right)(y)}{(x-y)^{i+1}},
$$

to indicate the "pole structure" in the "complex domain" $|x|>|y|$. The remaining "nonsingular parts" are not displayed since the pole terms determine the commutation relations of the components in Theorem 16 (iii)-(vii). We also note that $N$, the order of locality, determines the highest pole order.

3.2. Examples of Locality. Suppose that $U$ is a Lie algebra where for all $u, v, w \in U$ the commutator satisfies the Jacobi identity

$$
[[u, v], w]+[[v, w], u]+[[w, u], v]=0 .
$$


3.2.1. The Heisenberg Algebra. Consider the vector space

$$
\widehat{H}=\bigoplus_{n \in \mathbb{Z}} \mathbb{C} h_{n} \oplus \mathbb{C} K,
$$

with basis $h_{n}$ and central element $K$ obeying the Lie algebra commutation relations:

$$
\left[h_{m}, h_{n}\right]=m \delta_{m,-n} K, \quad\left[h_{m}, K\right]=0 .
$$

The formal series $h(z)=\sum_{n \in \mathbb{Z}} h_{n} z^{-n-1}$ obeys

$$
y^{-m}\left[h_{m}, h(y)\right]=y^{-m} \sum_{n \in \mathbb{Z}}\left[h_{m}, h_{n}\right] y^{-n-1}=K\left(\begin{array}{c}
m \\
1
\end{array}\right) y^{-1},
$$

a degree 1 polynomial in $m$. Thus Property (v) of Theorem 16 holds (with $\alpha=\beta=h$ ) which implies $h(z)$ is local of order $N=2$. Equivalently, we have Property (iii) of Theorem 16 with

$$
\left(h *_{0} h\right)(y)=0, \quad\left(h *_{1} h\right)(y)=K .
$$

Considering the Lie algebra components $h_{n}$ as being elements of the universal enveloping algebra of $\widehat{H}$ we obtain the OPE

$$
h(x) h(y) \stackrel{\text { OPE }}{\sim} \frac{K}{(x-y)^{2}} .
$$

This example is known in vertex algebra theory as the Heisenberg or free boson algebra and in conformal field theory as the bosonic string e.g. $[\mathrm{P}],[\mathrm{FMS}]$.

3.2.2. Affine Kac-Moody Algebras. Let $\mathfrak{g}$ be a finite dimensional Lie algebra with Lie bracket [, ] equipped with an invariant symmetric, bilinear form $\langle\rangle:, \mathfrak{g} \otimes \mathfrak{g} \rightarrow \mathbb{C}$ i.e.

$$
\langle[a, b], c\rangle=\langle a,[b, c]\rangle,
$$

for all $a, b, c \in \mathfrak{g}$. The affine Lie algebra or Kac-Moody algebra associated to $(\mathfrak{g},\langle\rangle$,$) is$ the vector space

$$
\widehat{\mathfrak{g}}=\mathfrak{g} \otimes \mathbb{C}\left[t, t^{-1}\right] \oplus \mathbb{C} K=\bigoplus_{n \in \mathbb{Z}} \mathfrak{g} \otimes t^{n} \oplus \mathbb{C} K,
$$

with Lie algebra commutators

$$
\begin{aligned}
& {\left[a \otimes t^{m}, b \otimes t^{n}\right]=[a, b] \otimes t^{m+n}+m\langle a, b\rangle \delta_{m,-n} K,} \\
& {\left[a \otimes t^{m}, K\right]=0,}
\end{aligned}
$$

for all $a, b \in \mathfrak{g}$. Define the formal series $a(z)=\sum_{n \in \mathbb{Z}} a_{n} z^{-n-1}$ for $a \in \mathfrak{g}$ where $a_{n}:=a \otimes t^{n}$. Then we find that for all $a, b \in \mathfrak{g}$

$$
y^{-m}\left[a_{m}, b(y)\right]=[a, b](y)+K\langle a, b\rangle\left(\begin{array}{c}
m \\
1
\end{array}\right) y^{-1} .
$$

This is Property (iii) of Theorem 16 for $N=2$ with

$$
\left(a *_{0} b\right)(y)=[a, b](y), \quad\left(a *_{1} b\right)(y)=K\langle a, b\rangle .
$$

Hence $a(z)$ and $b(z)$ are mutually local of order 2 if $\langle a, b\rangle \neq 0$, of order 1 if $\langle a, b\rangle=0$ and $[a, b] \neq 0$ and of order 0 if $\langle a, b\rangle=0$ and $[a, b]=0$. With a suitable universal enveloping algebra interpretation we obtain the OPE

$$
a(x) b(y) \stackrel{\mathrm{OPE}}{\sim} \frac{K\langle a, b\rangle}{(x-y)^{2}}+\frac{[a, b](y)}{x-y},
$$


This example is known as an affine Kac-Moody algebra theory or as a current algebra in conformal field theory. The Heisenberg algebra (41) corresponds to a 1dimensional subalgebra generated by $a$ for which $\langle a, a\rangle \neq 0$.

3.2.3. The Virasoro Algebra. Consider the vector space

$$
\text { Vir }=\bigoplus_{n \in \mathbb{Z}} \mathbb{C} L_{n} \oplus \mathbb{C} K,
$$

with basis $L_{n}$ and central element $K$ obeying the Virasoro algebra with commutation relations:

$$
\left[L_{m}, L_{n}\right]=(m-n) L_{m+n}+\frac{1}{2} K\left(\begin{array}{c}
m+1 \\
3
\end{array}\right) \delta_{m+n, 0}, \quad\left[L_{m}, K\right]=0 .
$$

(The factor of $\frac{1}{2}$ is conventional.) Define the formal series 5

$$
\omega(z)=\sum_{n \in \mathbb{Z}} L_{n} z^{-n-2} .
$$

Note that $\omega(z)$ is the formal series for a sequence with components

$$
\omega_{n}=L_{n-1} .
$$

Recalling the formal derivative (7) it follows that

$$
\begin{aligned}
y^{-m}\left[\omega_{m}, \omega(y)\right] & =\sum_{n \in \mathbb{Z}}\left[(m-n) \omega_{m+n-1}+\frac{1}{2} K\left(\begin{array}{c}
m \\
3
\end{array}\right) \delta_{m+n, 2}\right] y^{-m-n-1} \\
& =\partial \omega(y)+2 \omega(y)\left(\begin{array}{c}
m \\
1
\end{array}\right) y^{-1}+\frac{1}{2} K\left(\begin{array}{c}
m \\
3
\end{array}\right) y^{-3},
\end{aligned}
$$

a polynomial in $m$ of degree 3. Hence $\omega(z)$ is local of order 4 from Theorem 16 (v) and with a suitable universal enveloping algebra we obtain the OPE

$$
\omega(x) \omega(y) \stackrel{\text { OPE }}{\sim} \frac{\frac{1}{2} K}{(x-y)^{4}}+\frac{2 \omega(y)}{(x-y)^{2}}+\frac{\partial \omega(y)}{x-y} .
$$

\section{CReative Fields}

4.1. Fields. Let $V$ be a vector space over $\mathbb{C}$. We shall often refer to an element of $V$ as a state. Let $\operatorname{End}(V)$ denote the algebra of endomorphisms of $V$ i.e. linear maps from $V$ to $V$. $\operatorname{End}(V)$ is an associative algebra with unit given by the identity map $I_{V}$ over the field $\mathbb{C}$ with bilinear product given by the composition of linear maps $A B=A \circ B$ for all $A, B \in \operatorname{End}(V)$.

Consider a formal series $\alpha(z)=\sum_{n \in \mathbb{Z}} \alpha_{n} z^{-n-1}$ with components $\alpha_{n} \in \operatorname{End}(V)$. $\alpha(z)$ is called a field if for any $v \in V$

$$
\alpha_{n} v=0 \text { for } n \gg 0,
$$

i.e. for $n$ sufficiently large. The property (49) is called lower truncation and plays a vital role in vertex algebras.

\footnotetext{
${ }^{5} \omega(z)$ is usually notated by $T(z)$ in conformal field theory and is called the energy momentum tensor.
} 
For fields $\alpha(x), \beta(y)$ we may extend the definition of the bivariate formal series (29) to all $n \in \mathbb{Z}$ by defining

$$
C^{n}(\alpha(x), \beta(y))=(x-y)^{n} \alpha(x) \beta(y)-(-y+x)^{n} \beta(y) \alpha(x) .
$$

Clearly, this agrees with (29) for $n \geq 0$. In general, applying the formal expansion convention of (21) we find

$$
C^{n}(\alpha(x), \beta(y))=\sum_{l, m \in \mathbb{Z}} C_{l m}^{n}(\alpha, \beta) x^{-l-1} y^{-m-1},
$$

where for all $l, m, n \in \mathbb{Z}$

$$
C_{l m}^{n}(\alpha, \beta)=\sum_{i \geq 0}(-1)^{i}\left(\begin{array}{c}
n \\
i
\end{array}\right)\left(\alpha_{l+n-i} \beta_{m+i}-(-1)^{n} \beta_{m+n-i} \alpha_{l+i}\right) .
$$

Remark 19. By lower truncation, $C_{l m}^{n}(\alpha, \beta) v$ reduces to a finite sum of terms for each $v \in V$ and hence $C^{n}(\alpha(x), \beta(y))$ is a well-defined formal bivariate series.

Lemma 20. For all $k \geq 0$ and all $n \in \mathbb{Z}$ we have

$$
(x-y)^{k} C^{n}(\alpha(x), \beta(y))=C^{n+k}(\alpha(x), \beta(y)) .
$$

Proof. The expansion convention (21) implies $(x-y)^{k}(x-y)^{n}=(x-y)^{n+k}$ and $(x-y)^{k}(-y+x)^{n}=(-y+x)^{k}(-y+x)^{n}=(-y+x)^{n+k}$ for $k \geq 0$.

By Remark 19, for fields $\alpha(x), \beta(y)$ we may similarly extend the definition of the $n^{\text {th }}$ residue product $*_{n}$ to all $n \in \mathbb{Z}$ with

$$
\left(\alpha *_{n} \beta\right)(z)=\operatorname{Res}_{x} C^{n}(\alpha(x), \beta(z))=\sum_{i \geq 0}\left(\begin{array}{c}
n \\
i
\end{array}\right)\left((-z)^{i} \alpha_{n-i} \beta(z)-(-z)^{n-i} \beta(z) \alpha_{i}\right),
$$

with components

$$
\left(\alpha *_{n} \beta\right)_{m}=C_{0 m}^{n}(\alpha, \beta)=\sum_{i \geq 0}(-1)^{i}\left(\begin{array}{c}
n \\
i
\end{array}\right)\left(\alpha_{n-i} \beta_{m+i}-(-1)^{n} \beta_{m+n-i} \alpha_{i}\right) .
$$

Lemma 21. If $\alpha(z), \beta(z)$ are fields then $\left(\alpha *_{n} \beta\right)(z)$ is a field for all $n \in \mathbb{Z}$.

Proof. (54) implies $\left(\alpha *_{n} \beta\right)(z)$ is a field provided $C_{0 m}^{n}(\alpha, \beta) v=0$ for any $v \in V$ for $m \gg 0$. But $\beta_{m+i} v=0$ and $\beta_{m+n-i} \alpha_{i} v=0$ for $m \gg 0$ for some $v$ dependent finite range of $i$ following Remark 19 .

For $n<0,\left(\alpha *_{n} \beta\right)(z)$ is related to the normally ordered product (38) as follows:

Lemma 22. For fields $\alpha(x), \beta(z)$ and $k \geq 0$ we have

$$
\left(\alpha *_{-k-1} \beta\right)(z)=: \partial^{(k)} \alpha(z) \beta(z):
$$

Proof. The result follows directly from Lemma 13 .

Lemma 23. $\partial$ is a derivation of the $n^{\text {th }}$ residue product of two fields $\alpha(z), \beta(z)$ i.e.

$$
\partial\left(\alpha *_{n} \beta\right)(z)=\left(\partial \alpha *_{n} \beta\right)(z)+\left(\alpha *_{n} \partial \beta\right)(z) .
$$

Proof. From (50) we directly find

$$
\left(\partial_{x}+\partial_{z}\right) C^{n}(\alpha(x), \beta(z))=C^{n}(\partial \alpha(x), \beta(z))+C^{n}(\alpha(x), \partial \beta(z)) .
$$

Taking $\operatorname{Res}_{x}$, the result follows since $\operatorname{Res}_{x} \partial_{x} C^{n}(\alpha(x), \beta(z))=0$ from (9). 
The next theorem is fundamental to the theory of vertex algebras. It is often stated either as a foundational axiom [B, FLM, Li] or else is proved subject to some further assumed properties $[\mathrm{Li}, \mathrm{K}, \overline{\mathrm{MN}}]$. However, here we only assume that $\alpha(x), \beta(y)$ are local fields.

Theorem 24 (Borcherds-Frenkel-Lepowsky-Meurmann identity). Let $\alpha(x), \beta(y)$ be mutually local fields. Then for all $l, m, n \in \mathbb{Z}$ we have

$$
\sum_{i \geq 0}\left(\begin{array}{l}
l \\
i
\end{array}\right)\left(\alpha *_{n+i} \beta\right)_{l+m-i}=\sum_{i \geq 0}(-1)^{i}\left(\begin{array}{c}
n \\
i
\end{array}\right)\left(\alpha_{l+n-i} \beta_{m+i}-(-1)^{n} \beta_{m+n-i} \alpha_{l+i}\right) .
$$

Proof. Let $\alpha(z) \stackrel{N}{\sim} \beta(z)$ for $N \geq 0$. Thus (57) is the trivial identity $0=0$ for $n \geq N$ by locality and (32) so that we need only consider $n<N$. In a similar fashion to the proof of Theorem 16, the identity (57) is a consequence of Newton forward differences applied to an appropriate choice of sequence. Note that the right hand side of (57) is $C_{l m}^{n}(\alpha, \beta)$ of (51). For each $n<N$ we define a sequence $\gamma^{n}$ with components in $W:=\operatorname{End}(V)\left[\left[y, y^{-1}\right]\right]$ labelled by $l \in \mathbb{Z}$ as follows

$$
\left(\gamma^{n}\right)_{l}=y^{-l} \sum_{m \in \mathbb{Z}} C_{l m}^{n}(\alpha, \beta) y^{-m-1},
$$

with formal series

$$
\gamma^{n}(z)=y C^{n}(\alpha(y z), \beta(y)) .
$$

Since $\alpha(x) \stackrel{N}{\sim} \beta(y)$ and using (52) we find for each $n<N$ that

$$
(z-1)^{N-n} \gamma^{n}(z)=y^{1+n-N} C^{N}(\alpha(y z), \beta(y))=0 .
$$

Thus applying Newton's forward difference formula Theorem 10 (iii) we find

$$
\left(\gamma^{n}\right)_{l}=\sum_{i \geq 0}\left(\begin{array}{l}
l \\
i
\end{array}\right) R_{i}^{n}
$$

with $R_{i}^{n}$ for $i \geq 0$ given by

$$
\begin{aligned}
R_{i}^{n} & =y \operatorname{Res}_{z}(z-1)^{i} C^{n}(\alpha(y z), \beta(y)) \\
& =y^{1-i} \operatorname{Res}_{z} C^{n+i}(\alpha(y z), \beta(y)) \\
& =y^{-i} \operatorname{Res}_{x} C^{n+i}(\alpha(x), \beta(y))=y^{-i}\left(\alpha *_{n+i} \beta\right)(y),
\end{aligned}
$$

using (52) and that $\operatorname{Res}_{z} \rho(y z)=y^{-1} \operatorname{Res}_{x} \rho(x)$ for any formal series $\rho(x)$. We have therefore shown that $\left(\gamma^{n}\right)_{l} y^{l}$ is given by

$$
\sum_{m \in \mathbb{Z}} C_{l m}^{n}(\alpha, \beta) y^{-m-1}=\sum_{i \geq 0}\left(\begin{array}{l}
l \\
i
\end{array}\right) y^{l-i}\left(\alpha *_{n+i} \beta\right)(y) .
$$

The result follows on computing the coefficients of $y^{-m-1}$ in (59).

(57) specializes to the commutator formula Theorem 16 (iv) for $n=0$ and to the residue product formula (53) when $l=0$. There are a number of equivalent ways of writing (57). 
Proposition 25. Both of the following identities are equivalent to the Borcherds-FrenkelLepowsky-Meurmann identity:

(60)

$$
\sum_{i \geq 0} y^{-i-1} \delta^{(i)}\left(\frac{x}{y}\right)\left(\alpha *_{n+i} \beta\right)(y)=(x-y)^{n} \alpha(x) \beta(y)-(-y+x)^{n} \beta(y) \alpha(x),
$$

$$
y^{-1} \delta\left(\frac{x-z}{y}\right) \sum_{m \in \mathbb{Z}}\left(\alpha *_{m} \beta\right)(y) z^{-m-1}=z^{-1} \delta\left(\frac{x-y}{z}\right) \alpha(x) \beta(y)-z^{-1} \delta\left(\frac{-y+x}{z}\right) \beta(y) \alpha(x) .
$$

Proof. (59) is equivalent to

$$
C^{n}(\alpha(x), \beta(y))=\sum_{l \in \mathbb{Z}} x^{-l-1} \sum_{i \geq 0}\left(\begin{array}{l}
l \\
i
\end{array}\right) y^{l-i}\left(\alpha *_{n+i} \beta\right)(y) .
$$

Recalling (16) this can be written as (60). This in turn is equivalent to

$$
\begin{aligned}
& \sum_{n \in \mathbb{Z}} z^{-n-1} \sum_{i \geq 0} y^{-i-1} \delta^{(i)}\left(\frac{x}{y}\right)\left(\alpha *_{n+i} \beta\right)(y) \\
& =z^{-1} \sum_{n \in \mathbb{Z}}\left(\frac{x-y}{z}\right)^{n} \alpha(x) \beta(y)-z^{-1} \sum_{n \in \mathbb{Z}}\left(\frac{-y+x}{z}\right)^{n} \beta(y) \alpha(x) \\
& =z^{-1} \delta\left(\frac{x-y}{z}\right) \alpha(x) \beta(y)-z^{-1} \delta\left(\frac{-y+x}{z}\right) \beta(y) \alpha(x),
\end{aligned}
$$

recalling (12). Finally, Taylor's Theorem of Lemma 11 and (15) imply

$$
\delta\left(\frac{x-z}{y}\right)=\sum_{i \geq 0} \delta^{(i)}\left(\frac{x}{y}\right)\left(\frac{z}{y}\right)^{i}
$$

so that, after relabelling, we obtain

$$
\sum_{n \in \mathbb{Z}} z^{-n-1} \sum_{i \geq 0} y^{-i-1} \delta^{(i)}\left(\frac{x}{y}\right)\left(\alpha *_{n+i} \beta\right)(y)=y^{-1} \delta\left(\frac{x-z}{y}\right) \sum_{m \in \mathbb{Z}}\left(\alpha *_{m} \beta\right)(y) z^{-m-1} .
$$

Thus the result holds.

Remark 26. For $n \geq 0$ the Borcherds-Frenkel-Lepowsky-Meurmann identity (60) follows from locality using Theorem 16 (vi) and Lemma 7

The next result is very useful for the construction of local fields.

Lemma 27 (Dong's Lemma). Let $\alpha(z), \beta(z), \gamma(z)$ be mutually local fields. Then $\left(\alpha *_{n} \beta\right)(z)$ and $\gamma(z)$ are mutually local fields for all $n \in \mathbb{Z}$.

Proof. For some orders of locality $K, L, M \geq 0$ we have

$$
\alpha(z) \stackrel{K}{\sim} \beta(z), \quad \alpha(z) \stackrel{L}{\sim} \gamma(z), \quad \beta(z) \stackrel{M}{\sim} \gamma(z) .
$$

In particular, $C^{n}(\alpha(x), \beta(z))=0$ and $\left(\alpha *_{n} \beta\right)(z)=0$ for $n \geq K$. Hence we need only consider $n \leq K-1$. Let $N=K+L+M-n-1$ and define

$$
D(x, y, z)=(y-z)^{N}\left[\gamma(y), C^{n}(\alpha(x), \beta(z))\right] .
$$


Note that $N \geq 0$ since $L, M, K-n-1 \geq 0$. Using (52) we find

$$
\begin{aligned}
D(x, y, z) & =(y-z)^{M}(y-x+x-z)^{N-M}\left[\gamma(y), C^{n}(\alpha(x), \beta(z))\right] \\
& =(y-z)^{M} \sum_{r=0}^{K-n-1}\left(\begin{array}{c}
N-M \\
r
\end{array}\right)(y-x)^{N-M-r}\left[\gamma(y), C^{n+r}(\alpha(x), \beta(z))\right],
\end{aligned}
$$

where $r \leq K-n-1$ in the sum since $C^{n+r}(\alpha(x), \beta(z))=0$ for $n+r \geq K$. Therefore $N-M-r \geq L$ for each $r$ in the sum so that

$$
(y-z)^{M}(y-x)^{N-M-r}\left[\gamma(y), C^{n+r}(\alpha(x), \beta(z))\right]=0,
$$

since $\alpha(z) \stackrel{L}{\sim} \gamma(z)$ and $\beta(z) \stackrel{M}{\sim} \gamma(z)$. Thus $D(x, y, z)=0$ which implies

$$
C^{N}\left(\gamma(y),\left(\alpha *_{n} \beta\right)(z)\right)=\operatorname{Res}_{x} D(x, y, z)=0,
$$

i.e. $\gamma(z) \sim\left(\alpha *_{n} \beta\right)(z)$ with order of locality at most $N$.

4.2. Creative Fields. Let $1 \in V$ denote a distinguished state called the vacuum vector 6 A creative field for $a \in V$ is a field which we notate by

$$
a(z)=\sum_{n \in \mathbb{Z}} a_{n} z^{-n-1},
$$

with components or modes $a_{n} \in \operatorname{End}(V)$ such that

$$
\begin{aligned}
a_{-1} \mathbf{1} & =a, \\
a_{n} \mathbf{1} & =0, \text { for all } n \geq 0 .
\end{aligned}
$$

(63) is equivalent to $a(z)+\mathbf{1}=0$ (cf. (23)). We sometimes write (62) and (63) together as 7

$$
a(z) \mathbf{1}=a+O(z) \in V[[z]],
$$

where $V[[z]]$ is the space of formal power series in $z$ with coefficients in $V$.

The following lemma describes several important examples of creative fields.

Lemma 28. Let $a(z), b(z)$ be creative fields for states $a, b \in V$, respectively.

(i) $z a(z)$ creates the zero vector 0 ,

(ii) $I(z)=\operatorname{Id}_{V}$, the identity $V$ endomorphism, creates the vacuum $\mathbf{1}$,

(iii) $\lambda a(z)+\mu b(z)$ creates $\lambda a+\mu b$ for $\lambda, \mu \in \mathbb{C}$,

(iv) $\left(a *_{n} b\right)(z)$ creates $a_{n} b$ for $n \in \mathbb{Z}$,

(v) : $\partial^{(k)} a(z) b(z):$ creates $a_{-k-1} b$ for $k \geq 0$,

(vi) $\partial^{(k)} a(z)$ creates $a_{-k-1} \mathbf{1}$.

Proof. (i)-(iii) are trivially true. (53) implies that

$$
\begin{aligned}
\left(a *_{n} b\right)(z) \mathbf{1} & =\sum_{i \geq 0}\left(\begin{array}{c}
n \\
i
\end{array}\right)\left((-z)^{i} a_{n-i} b(z)-(-z)^{n-i} b(z) a_{i}\right) \mathbf{1} \\
& =\sum_{i \geq 0}\left(\begin{array}{c}
n \\
i
\end{array}\right)(-z)^{i} a_{n-i}(b+O(z))=a_{n} b+O(z),
\end{aligned}
$$

using creativity of $a(z)$ and $b(z)$. Hence (iv) holds. (iv) implies (v) on using Lemma (22). (vi) follows from (ii) and (v) on choosing $b=1$ and $b(z)=I(z)$.

\footnotetext{
${ }^{6}$ The vacuum vector is usually denoted by $|0\rangle$ in CFT.

7 This is usually written in CFT as $\lim _{z \rightarrow 0} a(z)|0\rangle=a$.
} 
Remark 29. A creative field $a(z)$ for $a \in V$ is clearly not unique since, by Lemma 28 (i), $a(z)+z b(z)$ also creates a for any creative field $b(z)$.

The lower truncation property (49) is refined for local creative fields as follows:

Corollary 30 (Lower Truncation). Let $a(z), b(z)$ be local creative fields for $a, b \in V$ respectively. Then $a(z) \stackrel{N}{\sim} b(z)$ implies

$$
a_{n} b=0 \text { for all } n \geq N \text {. }
$$

Proof. $\left(a *_{n} b\right)(z)=0$ for $n \geq N$ by (32) so that Lemma 28 (v) implies the result.

\section{Vertex Algebras}

5.1. Uniqueness and Translation Covariance. Consider a vector space $V$ with vacuum vector $1 \in V$ and a set of mutually local creative fields $\mathcal{F}:=\{a(z): a \in V\}$. By Remark 29, $a(z) \in \mathcal{F}$ is not the unique creative field for $a \in V$.

Proposition 31. Suppose that $\phi(z) \in \mathcal{F}$ is a creative field for the zero state 0 . Then

$$
\phi(z) \mathbf{1}=0 \Leftrightarrow \phi(z)=0 .
$$

Proof. Assume that $\phi(z) \mathbf{1}=0$. Let $a \in V$ with a creative field $a(z) \in \mathcal{F}$ where $a(z) \stackrel{N}{\sim} \phi(z)$ for some $N \geq 0$. Then

$$
0=x^{-N} C^{N}(\phi(x), a(y)) \mathbf{1}=x^{-N}(x-y)^{N} \phi(x) a(y) \mathbf{1}=\phi(x) a+O(y),
$$

i.e. $\phi(x) a=0$. This is true for any $a \in V$ so that $\phi(x)=0$. The converse is trivial.

This result immediately implies:

Corollary 32. Let $a(z), \widetilde{a}(z) \in \mathcal{F}$ be creative fields for $a \in V$. Then

$$
a(z)=\widetilde{a}(z) \Leftrightarrow a(z) \mathbf{1}=\widetilde{a}(z) \mathbf{1} .
$$

We now describe a uniqueness criterion for $\mathcal{F}$. Let $T \in \operatorname{End}(V)$ such that

$$
\begin{aligned}
T \mathbf{1} & =0, \\
{[T, a(z)] } & =\partial a(z) \text { for all } a(z) \in \mathcal{F} .
\end{aligned}
$$

In terms of modes, (67) is equivalent to

$$
\left[T, a_{n}\right]=-n a_{n-1} .
$$

$T$ is called a translation operator and $\mathcal{F}$ is said to be translation covariant if (66) and (67) are satisfied for a translation operator $T$.

Theorem 33 (Uniqueness). Let $\mathcal{F}$ be a set of mutually local creative fields for $V$. The elements of $\mathcal{F}$ are unique if and only if $\mathcal{F}$ is translation covariant.

Proof. Assume that the elements of $\mathcal{F}$ are unique. Define $T \in \operatorname{End}(V)$ by

$$
T a=a_{-2} \mathbf{1},
$$

for each $a \in V$ with unique creative field $a(z)$. By Lemma28(ii) we know that $I(z)=$ $\mathrm{Id}_{V}$ is a creative field for 1 and is therefore unique by assumption. Thus (69) implies (66). By Dong's Lemma 27 and Lemma 28 (iv) we also know that $\left(a *_{n} b\right)(z) \in \mathcal{F}$ is a creative field for $a_{n} b$ for each $a, b \in V$. Hence, by the assumed uniqueness property

$$
\left(a_{n} b\right)(z)=\left(a *_{n} b\right)(z) .
$$


In particular, using (54) we find that for all $a, b \in V$

$$
\begin{aligned}
T\left(a_{n} b\right)=\left(a_{n} b\right)_{-2} \mathbf{1} & =\sum_{i \geq 0}(-1)^{i}\left(\begin{array}{c}
n \\
i
\end{array}\right)\left(a_{n-i} b_{i-2}-(-1)^{n} b_{n-i-2} a_{i}\right) \mathbf{1} \\
& =a_{n} b_{-2} \mathbf{1}-n a_{n-1} b_{-1} \mathbf{1}=a_{n} T b-n a_{n-1} b .
\end{aligned}
$$

Hence $\mathcal{F}$ is translation covariant using (68).

Conversely, assume that $\mathcal{F}$ is translation covariant with some translation operator $T$. Thus for $a(z) \in \mathcal{F}$, (66) and (67) imply that $T a_{-k} \mathbf{1}=k a_{-k-1} \mathbf{1}$ for all $k \in \mathbb{Z}$. Hence $T^{n} a=T^{n} a_{-1} \mathbf{1}=n ! a_{-n-1} \mathbf{1}$ for all $n \geq 0$ so that

$$
a(z) \mathbf{1}=e^{z T} a \text {. }
$$

But if $\widetilde{a}(z)$ is another translation covariant creative field for $a$ then $\widetilde{a}(z) \mathbf{1}=e^{z T} a=$ $a(z) 1$. Hence by Corollary 32 we conclude that $a(z)=\widetilde{a}(z)$. Therefore the elements of $\mathcal{F}$ are unique.

5.2. Vertex Algebras. We have now gathered all the requisite concepts to define a vertex algebra. Let $Y(a, z)$ denote the unique translation covariant creative field for $a \in V$ of Theorem 33. $Y(a, z)$ is called the vertex operator for $a$. $Y$ can also be construed as a mapping

$$
\begin{aligned}
Y: V & \rightarrow \operatorname{End}(V)\left[\left[z, z^{-1}\right]\right], \\
a & \mapsto Y(a, z)=\sum_{n \in \mathbb{Z}} a_{n} z^{-n-1},
\end{aligned}
$$

called the state-field correspondence.

Definition 34. A Vertex Algebra consists of the data $(V, Y, T, 1)$ where $V$ is a vector space, a distinguished vacuum vector $1 \in V$, a translation operator $T \in \operatorname{End}(V)$ and a state-field correspondence $Y$ with the following properties:

locality: $Y(a, z) \sim Y(b, z)$ for all $a, b \in V$

creativity: $Y(a, z) \mathbf{1}=a+O(z)$,

translation covariance: $[T, Y(a, z)]=\partial Y(a, z), \quad T \mathbf{1}=0$.

Lemma 35. The state-field correspondence is an injective linear map.

Proof. Linearity follows from Lemma 28 (iii). Suppose $Y(a, z)=Y(b, z)$ for $a, b \in V$. Then $a_{-1}=b_{-1}$ so that $a=a_{-1} \mathbf{1}=b_{-1} \mathbf{1}=b$. Hence $Y$ is injective.

We describe a number of important properties of vertex operators:

Proposition 36. Let $a(z)=Y(a, z)$ and $b(z)=Y(b, z)$ be the vertex operators for $a, b \in V$.

(i) $Y(\mathbf{1}, z)=\operatorname{Id}_{V}$,

(ii) $Y(a, z) \mathbf{1}=e^{z T} a$,

(iii) $Y\left(a_{n} b, z\right)=\left(a *_{n} b\right)(z)$ for all $n \in \mathbb{Z}$,

(iv) $Y(T a, z)=\partial Y(a, z)$.

Proof. $\operatorname{Id}_{V} \in \mathcal{F}$ creates 1 , by Lemma 28 (ii), and is translation covariant giving (i). Property (ii) was shown in (71) in the proof of the Uniqueness Theorem 33 , $\left(a *_{n} b\right)(z) \in \mathcal{F}$ is a local creative field for $a_{n} b$ by Lemma 21 and Lemma 28 (iii). Translation covariance of $a(z)$ and $b(z)$ implies

$$
\left[T, C^{n}(a(x), b(z))\right]=C^{n}(\partial a(x), b(z))+C^{n}(a(x), \partial b(z))
$$


so that

$$
\begin{aligned}
{\left[T,\left(a *_{n} b\right)(z)\right] } & =\operatorname{Res}_{x}\left[T, C^{n}(a(x), b(z))\right] \\
& =\left(\partial a *_{n} b\right)(z)+\left(a *_{n} \partial b\right)(z)=\partial\left(a *_{n} b\right)(z),
\end{aligned}
$$

by Lemma 23. Thus $\left(a *_{n} b\right)(z)$ is translation covariant and so (iii) holds.

Lemma 15 and Lemma 28 (vi) imply $\partial Y(a, z)$ is a local creative field for $a_{-2} \mathbf{1}=$ $T a$. Translation covariance for $Y(a, z)$ implies

$$
[T, \partial Y(a, z)]=\partial[T, Y(a, z)]=\partial(\partial Y(a, z)),
$$

so that $\partial Y(a, z)$ is also translation covariant. Therefore (iv) follows from the Uniqueness Theorem 33 .

Corollary 37. $T$ is a derivation of the vertex algebra where for all $a, b \in V$ :

$$
T\left(a_{n} b\right)=(T a)_{n} b+a_{n} T b .
$$

Proof. $(T a)_{n}=-n a_{n-1}$ from Proposition 36 (iii). (68) implies $\left[T, a_{n}\right] b=(T a)_{n} b$.

Proposition 36 (iii) implies that, for a vertex algebra, we may replace all $n^{\text {th }}$ residue products $\left(a *_{n} b\right)(z)$ by the unique vertex operator $Y\left(a_{n} b, z\right)$ in the previous sections. Thus the locality Theorem 16 implies the Commutator Formulas

$$
\begin{aligned}
{\left[a_{m}, Y(b, z)\right] } & =\sum_{i \geq 0}\left(\begin{array}{c}
m \\
i
\end{array}\right) Y\left(a_{i} b, z\right) z^{m-i}, \\
{\left[a_{m}, b_{n}\right] } & =\sum_{i \geq 0}\left(\begin{array}{c}
m \\
i
\end{array}\right)\left(a_{i} b\right)_{m+n-i},
\end{aligned}
$$

for all $m, n \in \mathbb{Z}$. Similarly (54) implies the Associator Formula

$$
\left(a_{n} b\right)_{m}=\sum_{i \geq 0}(-1)^{i}\left(\begin{array}{l}
n \\
i
\end{array}\right)\left(a_{n-i} b_{m+i}-(-1)^{n} b_{m+n-i} a_{i}\right)
$$

These can be combined into the Borcherds-Frenkel-Lepowsky-Meurmann identity

$$
\sum_{i \geq 0}\left(\begin{array}{l}
l \\
i
\end{array}\right)\left(a_{n+i} b\right)_{l+m-i}=\sum_{i \geq 0}(-1)^{i}\left(\begin{array}{l}
n \\
i
\end{array}\right)\left(a_{l+n-i} b_{m+i}-(-1)^{n} b_{m+n-i} a_{l+i}\right)
$$

for all $l, m, n \in \mathbb{Z}$ (cf. (57)). This in turn is equivalent to (cf. (61))

$$
\begin{aligned}
& z^{-1} \delta\left(\frac{x-y}{z}\right) Y(a, x) Y(b, y)-z^{-1} \delta\left(\frac{-y+x}{z}\right) Y(b, y) Y(a, x) \\
& =y^{-1} \delta\left(\frac{x-z}{y}\right) Y(Y(a, z) b, y) .
\end{aligned}
$$

(75) and (76) are axioms in the original formulation of vertex algebras by Borcherds in [B]. These were shown to be equivalent to the identity (78), called the Jacobi identity by Frenkel, Lepowsky and Meurmann [FLM $]$. 


\subsection{Translation and Skewsymmetry.}

Lemma 38 (Translation Symmetry). T is a generator of translation symmetry:

$$
e^{y T} Y(a, x) e^{-y T}=Y(a, x+y) .
$$

Proof. The Baker-Campbell-Hausdorff formula for linear operators $A, B$ states that

$$
e^{A} B e^{-A}=e^{\operatorname{ad}_{A}} B
$$

where $\operatorname{ad}_{A}(\cdot)=[A, \cdot]$ is the adjoint operator. Thus we find

$$
e^{y T} Y(a, x) e^{-y T}=e^{y \operatorname{ad}_{T}} Y(a, x)=e^{y \partial} Y(a, x),
$$

by translation covariance (67). The result follows from Taylor's theorem (24).

Lemma 39 (Skew-Symmetry). Let $a, b \in V$, a vertex algebra. Then

$$
Y(a, z) b=e^{z T} Y(b,-z) a,
$$

or in terms of components:

$$
a_{n} b=(-1)^{n+1} \sum_{k \geq 0}(-1)^{k} T^{k} b_{n+k} a .
$$

Proof. Let $Y(a, z) \stackrel{N}{\sim} Y(b, z)$ so that

$$
(z-y)^{N} Y(a, z) Y(b, y) \mathbf{1}=(z-y)^{N} Y(b, y) Y(a, z) \mathbf{1} .
$$

By Proposition 36 (ii) and translation symmetry we have

$$
\begin{aligned}
(z-y)^{N} Y(a, z) e^{y T} b & =(z-y)^{N} Y(b, y) e^{z T} a \\
& =(z-y)^{N} e^{z T} Y(b, y-z) a .
\end{aligned}
$$

Lower truncation (64) implies $x^{N} Y(b, x) a$ contains no negative powers of $x$. Thus $(z-y)^{N} Y(b, y-z) a$ also contains no negative powers of $y$. Taking $y=0$ in (81) we obtain (79) on multiplying by $z^{-N}$. (80) follows immediately.

5.4. Examples of Vertex Algebras. We have the following very useful generating theorem [FKRW], [MP].

Theorem 40 (Generating Theorem). Let $V$ be a vector space with $1 \in V$ and $T \in$ $\operatorname{End}(V)$. Let $\left\{a^{i}(z)\right\}_{i \in \mathcal{I}}$ for some indexing set $\mathcal{I}$ be a set of mutually local, creative, translation-covariant fields which generates $V$ i.e.

$$
V=\operatorname{span}\left\{a_{n_{1}}^{i_{1}} \ldots a_{n_{k}}^{i_{k}} \mathbf{1} \mid n_{1}, \ldots, n_{k} \in \mathbb{Z}, i_{1}, \ldots i_{k} \in \mathcal{I}\right\}
$$

Then there is a unique vertex algebra $(V, Y, 1, T)$ with vertex operators defined on the spanning set by

$$
Y\left(a_{n_{1}}^{i_{1}} \ldots a_{n_{k}}^{i_{k}} \mathbf{1}, z\right)=a^{i_{1}} *_{n_{1}}\left(a^{i_{1}} *_{n_{2}}\left(\ldots\left(a^{i_{k}} *_{n_{k}} I\right)\right)\right)(z),
$$

a composition of $k$ residue products and where $I(z)=Y(\mathbf{1}, z)=\operatorname{Id}_{V}$.

Proof. $\mathcal{F}=\left\{a^{i_{1}} *_{n_{1}}\left(a^{i_{1}} *_{n_{2}}\left(\ldots\left(a^{i_{k}} *_{n_{k}} I\right)\right)\right)(z)\right\}$ is a set of mutually local creative fields for $V$ by repeated use of Lemma 21, Dong's Lemma 27 and Lemma 28 (iv). Furthermore, $a^{i_{1}} *_{n_{1}}\left(a^{i_{1}} *_{n_{2}}\left(\ldots\left(a^{i_{k}} *_{n_{k}} I\right)\right)\right)(z)$ is translation covariant by (73). Hence, by the Uniqueness Theorem $33, \mathcal{F}$ forms a set of unique vertex operators on the spanning set and therefore by linearity on $V$. 
5.4.1. The Heisenberg Vertex Algebra. The Heisenberg vertex algebra is constructed from the Verma module $8 M_{0}$ of the Heisenberg Lie algebra (41) given by

$$
M_{0}=\operatorname{span}\left\{h_{-n_{1}} \ldots h_{-n_{k}} v_{0} \mid n_{1}, \ldots, n_{k} \geq 1\right\},
$$

where $h_{n} v_{0}=0$ for all $n \geq 0$ and $K v_{0}=v_{0}$. Then with $V=M_{0}$ and $1=v_{0}$ we find $h(z)$ is a creative field for $h=h_{-1} \mathbf{1}$ which is translation covariant for

$$
T=\sum_{n \geq 0} h_{-n-1} h_{n} .
$$

Thus Theorem 40 and Lemma 28 imply that $h(z)$ generates a vertex algebra with

$$
Y\left(h_{-n_{1}} \ldots h_{-n_{k}} \mathbf{1}, z\right)=: \partial^{\left(n_{1}\right)} h(z): \partial^{\left(n_{2}\right)} h(z) \ldots: \partial^{\left(n_{k-1}\right)} h(z) \partial^{\left(n_{k}\right)} h(z):: \ldots:,
$$

for $n_{1}, \ldots, n_{k} \geq 1$.

5.4.2. The Virasoro Vertex Algebra. The Virasoro vertex algebra is constructed from a Verma module $M_{C, 0}$ of the Virasoro Lie algebra (46) defined by

$$
M_{C, 0}=\operatorname{span}\left\{L_{-n_{1}} \ldots L_{-n_{k}} v_{0} \mid n_{1}, \ldots, n_{k} \geq 1\right\}
$$

where $L_{n} v_{0}=0$ for all $n \geq 0$ and $K v_{0}=C v_{0}$. Then $\omega(z)=\sum_{n \in \mathbb{Z}} L_{n} z^{-n-2}$ is translation covariant for $T=L_{-1}$ but is not a creative field with vacuum $v_{0}$ since

$$
\omega(z) v_{0}=z^{-1} L_{-1} v_{0}+L_{-2} v_{0}+O(z) .
$$

But since $L_{1} L_{-1} v_{0}=0$ it follows that

$$
M_{C, 1}=\operatorname{span}\left\{L_{-n_{1}} \ldots L_{-n_{k}} L_{-1} v_{0} \mid n_{1}, \ldots, n_{k} \geq 1\right\},
$$

is submodule of $M_{C, 0}$. Abusing notation by identifying states, operators and fields associated with $M_{C, 0}$ with the corresponding states, operators and fields induced on the quotient Vir $=M_{C, 0} / M_{C, 1}$ we find that $Y(\omega, z)=\omega(z)$ generates a vertex algebra with $T=L_{-1}, \mathbf{1}=v_{0}$ and $V=$ Vir with vertex operators

$$
Y\left(L_{-n_{1}} \ldots L_{-n_{k}} \mathbf{1}, z\right)=: \partial^{\left(n_{1}\right)} L(z): \partial^{\left(n_{2}\right)} L(z) \ldots: \partial^{\left(n_{k-1}\right)} L(z) \partial^{\left(n_{k}\right)} L(z):: \ldots: \text {, }
$$

for $n_{1}, \ldots, n_{k} \geq 2$.

\section{REFERENCES}

[B] R. Borcherds, Vertex algebras, Kac-Moody algebras, and the Monster, Proc. Nat. Acad. Sc. 83 (1986) 3068-3071.

[BPZ] A. Belavin, A. Polyakov and A. Zamolodchikov, Infinite conformal symmetry in twodimensional quantum field theory, Nucl. Phys. B241 (1984) 333-380.

[FHL] I. Frenkel, J. Lepowsky and Y.-Z. Huang, On Axiomatic Approaches to Vertex Operator Algebras and Modules, Mem. AMS. 104 No. 4941993.

[FKRW] E. Frenkel, V. Kac, A. Radul and W. Wang, $W_{1+\infty}$ and $W\left(g l_{\infty}\right)$ with central charge $N$, Comm. Math. Phys. 170 (1995) 337-357.

[FLM] I. Frenkel, J. Lepowsky and A. Meurman, Vertex Operator Algebras and the Monster, Academic Press, Boston, 1988.

[FMS] P. Di Francesco, P. Mathieu, and D. Senechal, Conformal Field Theory, Springer Graduate Texts in Contemporary Physics, Springer-Verlag, New York (1997).

[G] P. Goddard, Meromorphic conformal field theory, Infinite Dimensional Lie Algebras and Groups, Proc. Conf. at CIRM, Luminy, World Sci., Singapore, 1989.

[K] V. Kac, Vertex Algebras for Beginners, Univ. Lect. Ser. 10, AMS, 1998.

[Li] H. Li, Local systems of vertex operators, J. Pure Appl. Alg. 109 (1996) 143-195.

[LL] J. Lepowsky and H. Li, Introduction to Vertex Algebras, Progress in Math. Vol. 227, Birkhäuser, Boston, 2004.

$\overline{8 \text { e.g. See }[\mathrm{K}]}$, [MT] for further details 
[LZ] B.H. Lian and G.J. Zuckerman, Commutative quantum operator algebras, J. Pure Appl. Alg. 100, (1995) 117-140.

[MN] A. Matsuo and K. Nagatomo, Axioms for a Vertex Algebra and the Locality of Quantum Fields, Math. Soc. of Japan Memoirs Vol. 4, Tokyo, 1999.

[MT] G. Mason and M.P. Tuite, Vertex operators and modular forms, A Window into Zeta and Modular Physics, eds. K. Kirsten and F. Williams, Cambridge University Press, (Cambridge, 2010), MSRI Publications 57 (2010) 183-278.

[MP] A. Meurman and M. Primc, Vertex operator algebras and representations of affine Lie algebras, Acta Applic. Math. 44 (1996) 207-215.

[N] I. Newton, Philosophiæ Naturalis Principia Mathematica, Book III, London, 1687.

[P] J. Polchinski, String theory, Volume I, Cambridge University Press, Cambridge, 1998.

School of Mathematics, Statistics and Applied Mathematics, National University of IRELAND GALWAY, GALWAY, IRELAND

E-mail address: michael.tuite@nuigalway.ie 